\title{
Metabolic syndrome nephropathy (early-diagnosis of intrarenal arteriosclerotic lesions by renal ultrasonography)
}

\author{
Hiroki Tsuchida ${ }^{1)}$ and Kiyoaki Iwashita ${ }^{2}$
}

\begin{abstract}
:
The renal complication of type 2 diabetes mellitus is diagnosed as the diabetic nephropathy. Its pathophysiology has not yet been fully clarified, however. Although a concept of diabetic kidney disease has recently been proposed, the background of its pathophysiology is limited to type 2 diabetes and definite pathophysiological features are difficult to indicate. Under these conditions, we propose metabolic syndrome nephropathy (combined renal microangiopathy and intrarenal arteriosclerosis), taking account of the metabolic syndrome which plays an important role in the development of type 2 diabetes. This article is a report of our early-diagnosis method established for such nephropathy.

The new diagnostic method (using vascular resistance measurement by renal ultrasound imaging and estimated renal blood flow measurement with a contrast agent for ultrasound imaging), as compared with the conventional method (using albuminuria detection and estimated glomerular filtration rate measurement), showed a marked increase of diagnostic rate $(89.4 \%$ vs. $51.3 \%)$. This new method, if applicable in the early-diagnosis of renal damage, will hold promise in providing a clue for preventing the end-stage renal failure.
\end{abstract}

\section{Key words:}

Metabolic syndrome nephropathy, Diabetic nephropathy, Diabetic kidney disease, Resistive index, Estimated renal blood flow

\section{Background}

(1) Diabetic nephropathy (DN), a complication of type 2 diabetes mellitus (T2DM), is a primary renal disease leading to end-stage renal failure ${ }^{1)}$.

(2) As reported in the Steno 2 randomized trial ${ }^{2)}$ and JDOIT $^{3)}$, comprehensive treatment mainly targeted at hypertension, dyslipidemia and hyperglycemia is effective against T2DN, but it does not suffice.

(3) T2DM is deeply involved in the pathophysiological state of the metabolic syndrome (MetS) $)^{4,5)}$.

(4) We have named MetS-associated renal damage 'metabolic syndrome nephropathy' (MetS-N).

(5) The early diagnosis of MetS-N, and optimal strategies against MetS-N are therefore needed for preventing endstage renal failure.

\section{Objectives}

The aim of this article is to clarify the relationship between T2DM and MetS, and to evaluate whether the introduction of a new diagnostic method to MetS patients with $\mathrm{T}$ 2DM improves the diagnostic accuracy of MetS-N.

\section{Subjects and Methods}

\section{Definition of MetS-N}

Condition 1: Clinical features - to fit the definition of MetS in the Japanese people ${ }^{6}$. Obesity was defined as BMI $\geq 25$. No visceral fat area was estimated.

Condition 2: Pathological features - to have an intrarenal arteriosclerotic lesion (especially fibroelastosis centering on the intrarenal arteries). In this regard, however, it is very difficult to constantly get renal pathological sample. At present,

\footnotetext{
1) Research Institute of Strategy on Chronic Diseases, Association of Health-care and Medication for Labor in Chiba

2) Department of Clinical Laboratory, National Hospital Organization Saitama National Hospital

Corresponding author: Hiroki Tsuchida, h-tsuchida@min-iren-c.or.jp

Received: February 20, 2018, Accepted: April 19, 2018

Copyright (C) 2018 Japan Society for Vascular Failure
} 
therefore, such pathological features may be replaced with renal arterial resistive index (RI) and estimated renal blood flow (eRBF) by an ultrasound method as surrogate markers $^{4,5)}$.

Condition 3: Findings suggestive of renal damage- It is established by any of the following: proteinuria by urine test paper method; microalbuminuria ( $\geqq 30 \mathrm{mg} / \mathrm{g} \mathrm{Cr}$ ); and eGFR $\left(<60 \mathrm{~mL} / \mathrm{min} / 1.73 \mathrm{~m}^{2}\right)$.

The disorder that meets conditions 1 and 2 above is defined as MetS-N, and condition 3 is used as reference information.

\section{Relationship between T2DM and MetS (Retrospec- tive Study)}

Four hundred and sixty-five (465) patients (with a maleto-female ratio of 1:0.85) with proteinuria negative T2DM under ambulatory management were recruited to estimate the proportion of those who fulfill the MetS diagnosis criteria. Of these subjects, those with two or more MetS risk factors (obesity, hypertension, and dyslipidemia) except hyperglycemia were diagnosed as having MetS. Furthermore, the subjects were examined for the status of the use of drugs associated with such risk factors and those in whom any such drug was in use were regarded as having the risk factor in-

Table 1. Clinical characteristics of 465 proteinuria-negative T2DM

\begin{tabular}{lc}
\hline Number of Patients & 465 \\
Age (years old) & $60.6 \pm 7.4$ \\
eGFR $\left(\mathrm{mL} / \mathrm{min} / 1.73 \mathrm{~m}^{2}\right)$ & $78.7 \pm 17.3$ \\
ACR $(\mathrm{mg} / \mathrm{gCr})$ & $27.4 \pm 45.6$ \\
BMI & $24.9 \pm 4.0$ \\
BPS $(\mathrm{mmHg})$ & $130 \pm 15$ \\
BPD $(\mathrm{mmHg})$ & $75 \pm 10$ \\
TG $(\mathrm{mg} / \mathrm{dL})$ & $203.7 \pm 33.8$ \\
HDL-C $(\mathrm{mg} / \mathrm{dL})$ & $60.5 \pm 17.9$ \\
HbA1c $(\%)$ & $7.2 \pm 1.0$ \\
Duration od DM (years) & $10.9 \pm 7.6$ \\
\hline male:female=1:0.85 &
\end{tabular}

volved in the relevant drug. Their clinical characteristics were shown in Table 1.

Evaluation in the longitudinal analysis was made by confirming, at the time of medical examination, the presence of such a risk factor during the course of one month to 40 years as well as the condition described in Table 2.

In this estimation, cross-sectional analysis (analysis at the time of medical examination) and longitudinal analysis (risk factors for MetS are picked up from the past clinical data) are conducted to evaluate the closeness of relationship between T2DM and MetS.

\section{Progressive Pattern of MetS (Prospective Study)}

Of the 4946 medical examinees in small and mediumsized construction companies in year 2008, 77 with obesity alone are selected and followed up solely under the lifestyle guidance for 5 years. This study is intended to clarify the pattern of occurrence of hypertension, dyslipidemia, and hyperglycemia and in addition to measure the rate of occurrence of proteinuria by the urine test paper method.

\section{Diagnosis of MetS-N}

After other clinicopathological entity was excluded from clinical course, urinary findings, diagnostic imaging, and any information where possible, 135 male individuals (22 with proteinuria positive findings and 113 with proteinuria negative findings) with a full house of MetS risk factors (four factors: obesity, hypertension, dyslipidemia, and hyperglycemia) and aged 40-74 years are recruited (Figure 1). Of these subjects, 113 without proteinuria are examined for renal damage by the "albuminuria and eGFR" method and by the ultrasound "RI and eRBF" method. Their clinical characteristics were shown in Table 2.

(1) Renal damage determination by urinary findings and renal function (conventional method)

The subjects are classified according to the ACR and eGFR values into four categories (Groups A, B, C, and D) defined by ACR $\geqq 30 \mathrm{mg} / \mathrm{g} \mathrm{Cr}$ and eGFR $<60 \mathrm{~mL} / \mathrm{min} / 1.73$ $\mathrm{m}^{2}$. In addition, the category showing an ACR value $\geqq 300$

Table 2. Clinical characteristics of 135 full-house MetS

\begin{tabular}{lcccc}
\hline & G-ABCD & G-E & P-value & Level of significance \\
\hline Number of Patients & 113 & 22 & & \\
Age (years old) & $60.3 \pm 9.0$ & $62.9 \pm 10.2$ & 0.218 & $*$ \\
eGFR $\left(\mathrm{mL} / \mathrm{min} / 1.73 \mathrm{~m}^{2}\right)$ & $66.8 \pm 17.0$ & $40.9 \pm 19.1$ & 0.000 & $*$ \\
ACR $(\mathrm{mg} / \mathrm{gCr})$ & $36.8 \pm 51.5$ & $1564.0 \pm 1539.4$ & 0.000 & $*$ \\
BMImax & $28.6 \pm 3.8$ & $30.3 \pm 5.4$ & 0.154 & $*$ \\
BPSmax $(\mathrm{mmHg})$ & $171 \pm 17$ & $181 \pm 19$ & 0.014 & \\
BPDmax $(\mathrm{mmHg})$ & $104 \pm 10$ & $105 \pm 13$ & 0.701 & \\
TGmax $(\mathrm{mg} / \mathrm{dL})$ & $524.9 \pm 426.7$ & $445.1 \pm 350.3$ & 0.412 & \\
HDL-Cmin $(\mathrm{mg} / \mathrm{dL})$ & $41.9 \pm 12.2$ & $43.3 \pm 15.2$ & 0.615 & \\
HbA1cmax $(\%)$ & $8.9 \pm 2.5$ & $9.4 \pm 3.5$ & 0.563 & \\
FPGmax (mg/dL) & $202.9 \pm 80.0$ & $247.5 \pm 123.0$ & 0.156 & \\
Duration od DM (years) & $12.9 \pm 8.9$ & $11.5 \pm 7.4$ & 0.477 & \\
\hline
\end{tabular}

only male

${ }^{* *} \mathrm{P}<0.01{ }^{*} \mathrm{P}<0.05$ 




Subjects: Categorized 135 patients (male patients with a full house of MetS risk factors aged 40-74yrs.)

Figure 1.

Sampling point by Color Doppler on lobular Artery

a

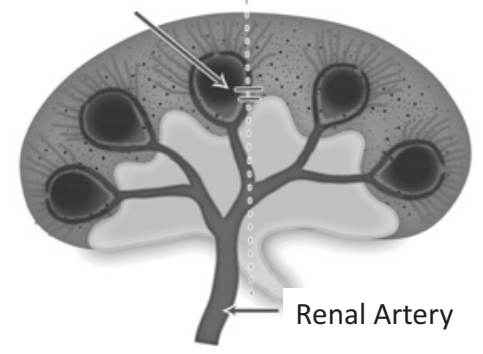

Figure 2. (a, b) The method of Resistive Index



A: peak systolic velocity

B: lowest diastolic velocity

Renal arterial resistive index(RI)

by Pulse Wave: $\mathrm{RI}=\mathrm{A}-\mathrm{B} / \mathrm{A}$ $\mathrm{mg} / \mathrm{g} \mathrm{Cr}$ or proteinuria positive defined as Group E, and the frequency of renal damage is then determined (Figure 1).

(i) ACR measurement ${ }^{7}$ : ACR is measured with an albumin kit (N-Assay TIA Micro Alb; Nittobo Medical Co., Ltd.) and classified into three grades: normoalbuminuria (<30 mg/g Cr); microalbuminuria (30-299 mg/g Cr); and macroalbuminuria ( $\geqq 300 \mathrm{mg} / \mathrm{g} \mathrm{Cr}$ ).

(ii) eGFR measurement ${ }^{8)}$ : An estimate equation for the Japanese people formulated from serum creatinine levels, sex, and age is adopted.

(2) Renal damage determination by ultrasound diagnosis (new method)

The subjects consisted of 20 normal individuals, 113(proteinuria negative) individuals with a full house of MetS risk factors, and 22(proteinuria positive) individuals with a full house of MetS risk factors, and thresholds were set at 0.650 for RI and 75 for eRBF.

(i) Renal arterial resistive index (RI) method $^{9-11)}$ (Figure 2):

While the subject is kept on bed rest in the supine position under fasting conditions, the kidney is visualized by frontal longitudinal scanning of flank. The interlobar arteries running along the renomedullary pyramids are then identi- fied by color Doppler imaging and the sampling point is constantly arranged at the position a little closer to the peripheral site from the interface between the central hyperechoic image and cortex. Measurement at each sampling point is made in triplicate at least to calculate RI as the peripheral vascular resistance. RI is calculated according to the formula: RI = (peak systolic velocity - lowest diastolic velocity) / (peak systolic velocity), as shown in Figure 2. The average of both kidneys is taken to obtain the RI value. Its normal value is defined as $<0.650$.

(ii) Estimated renal blood flow (eRBF) measurement with a contrast agent for ultrasound imaging (Figure 3):

While the subject is kept on bed rest in the supine position under fasting conditions, the kidney is visualized by frontal longitudinal scanning of flank.

After $2 \mathrm{~mL}$ of a contrast agent for ultrasound imaging $\left(\right.$ Sonazoid $^{\mathrm{TM}}$ ) is reconstituted with sterile water, the subject is intravenously injected with a volume, " $0.15 \times$ body weight $(\mathrm{kg})$ " $\mathrm{mL}$, of the reconstituted solution via an left median vein and flushed with $3 \mathrm{~mL}$ of saline (for 3-5 seconds). Recording is made from the time when the segmental artery of the kidney glittered with the contrast agent (it takes 


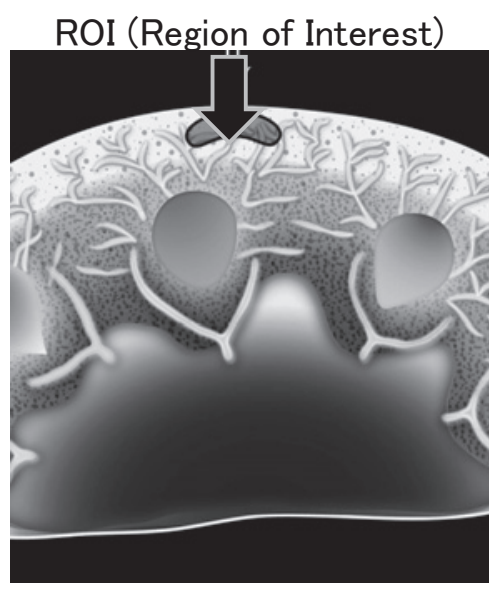

Sonazoid ${ }^{\mathrm{TM}}$ accumulated in the region of interest (ROI) is used for measurement.

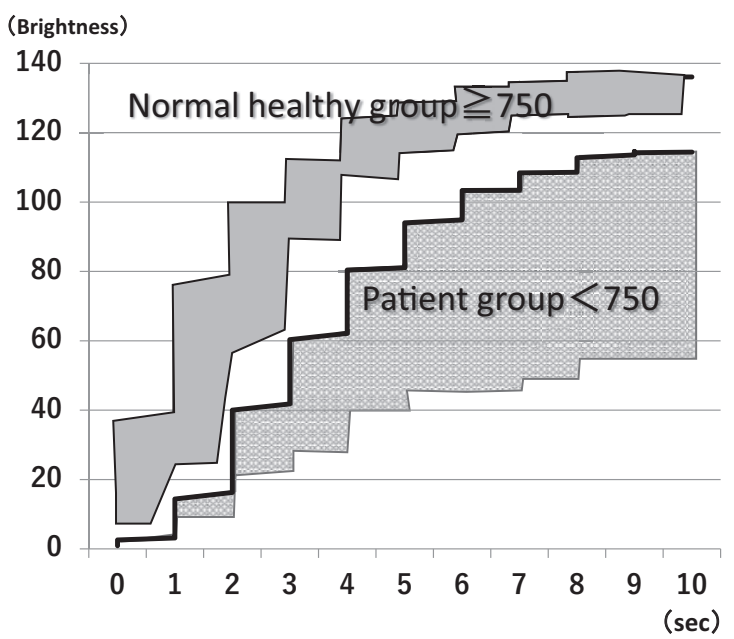

The brightness curve (TIC) for each of the normal healthy group and patient group. The eRBF was integrated the area under the TIC.

Figure 3. about 10-odd seconds) up to the renal cortex thoroughly glittered. After the completion of examination, the region of interest (ROI; the number of pixels is adjusted to 1000) is established near the capsule of the renal cortex and Sonazoid $^{\mathrm{TM}}$ reaching the ROI is counted for 10 seconds to prepare the TIC (time intensity curve). The area under the TIC (eRBC) was obtained from the following:20 normal individuals (gray area) and 113 urinary protein-negative patients with a full house of MetS risk factors (red area). Use is made of a Hitachi Ascendus ultrasound system (Hitachi, Ltd.) and a probe (5-1 MHz), and the MI value is set at 0.2. The present measurement is the world's first attempt and it is, therefore, desirable that fundamental verification be conducted in the future. The normal value is defined as $\geqq 750$. The rationale for threshold selection is explained from the fact that 0.650 (or, in some reports, 0.700 ) has already been reported as the optimum value suggestive of renal function and pathological features by many investigators with respect to RI. For eRBF, on the other hand, its appropriate threshold is nothing short of ignorance in view of unprecedented experiment. This will be the subject of further study. In the present study, however, a threshold value of 750 was selected temporarily.

\section{Confirmation of the Progressive Pattern of Renal Damage}

In the groups in which individuals are classified according to the level of ACR and eGFR, the rate of occurrence of RI or eRBF abnormalities is determined to evaluate the directionality of progression of renal damage.

(NOTE) The renal blood flow measurement with Sonazoid $^{\mathrm{TM}}$ has received prior Chiba Kensei Hospital institutional review board approval. Before examination, all participants have been checked for allergies and granted written informed consent. As of January 2018, 356 subjects have un- dergone examination and no adverse effects have been developped.

\section{Results}

\section{Relationship between T2DM and MetS (Retrospec- tive Study)}

(1) According to the cross-sectional diagnosis (done with information available at the time of medical examination) shown in Figure 4, the probability that T2DM patients will have MetS is $40 \%$, whereas the corresponding value at the longitudinal diagnosis (done with information including the past data) is $74.2 \%$. This indicates that longitudinal diagnosis, which takes into account a long-term clinical course, is needed in evaluating the relationship of MetS patients to T2 DM.

(2) The clinical pattern (Figure 5) shows progression from obesity through hypertension and dyslipidemia to hyperglycemia - this is known as 'metabolic domino.' During this process, the incidence of proteinuria suggestive of renal damage increases.

\section{Frequency and Trend of MetS-N}

(1) The proteinuria positive group (Group E) of subjects can be diagnosed as having renal damage and their number stood at 22 .

(2) In 113 subjects of the proteinuria negative groups (Groups A, B, C, and D), renal damage can be detected through the approach given below. First, according to the conventional method ${ }^{12)}$ (diagnostic method using ACR and eGFR) currently available in Japan, Groups B, C, and D of subjects can be diagnosed as having renal damage with diagnostic rates of $16.8 \%, 16.8 \%$, and $17.7 \%$, respectively, (51.3\% in total). Second, the relevant diagnostic rate in the 


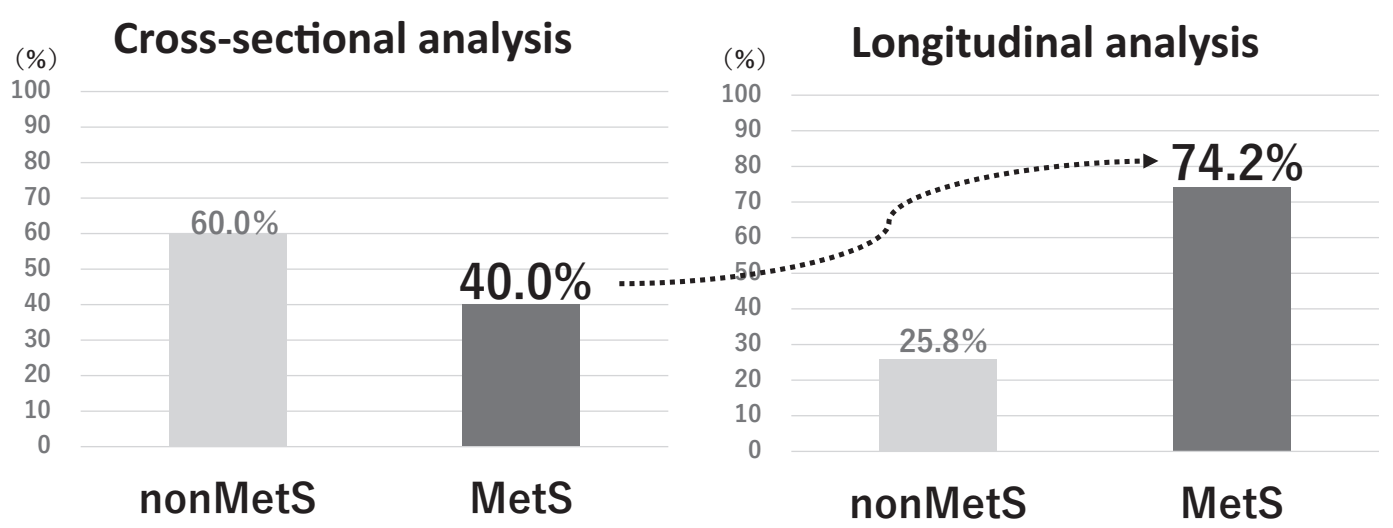

Retrospective Analysis: Percentage of metabolic syndrome (MetS) in T2DM (subjects: 465 patients with proteinuria negative T2DM)

MetS: metabolic syndrome, nonMetS : non-metabolic syndrome

Figure 4.


Prospective Analysis: Individuals with only obesity in medical examination for metabolic syndrome--5years' progress subjects : 77 obese individuals out of the 4,946 physical examinees in small and midium-sized construction companies in year 2008

Obesity (BMI $\geqq 25$ ); Hypertension (BP $\geqq 130$ \&/or $85 \mathrm{mmHg}$; Dyslipidemia ( $T G \geqq 150$ \&/or HDL-C $<40 \mathrm{mg} / \mathrm{dL}$ ) ; Hyperglycemia (HbA1c [NGSP] equivalent value $\geqq 5.8 \%$ )

Figure 5.

new method stood at $80.5 \%$ when calculated from RI abnormalities $\geqq 0.650$ and $79.6 \%$ when from eRBF abnormalities $<750$. When these abnormalities were combined together, a diagnostic rate of $89.4 \%$ was obtained totally. Each data were as follows: G-A, 78\% (43/55 people); G-B, $84 \%$ (16/ 19 people); G-C, $89 \%$ (17/19 people); G-D, 95\% (19/20 people); and G-E, 95\% (18/19 people) (Figure 6; large solid circle, normal; small open circle, abnormal). The new method shows 1.74 times the diagnostic rate in the conventional method, producing a marked improvement in diagnostic accuracy.

(3) Directionality of renal damage progression

$\mathrm{RI}$ and $\mathrm{eRBF}$ as surrogate markers of intrarenal arteriosclerotic lesions, although their results are not exactly equivalent to each other, displayed an outstanding mirror image when subjects were divided into three groups: 'normal,' 'proteinuria negative MetS-group' and 'proteinuria positive MetS-group,' i.e., a striking similarity was shown between them as surrogate markers (Figure 7).
Furthermore, as shown in Figure 6, the probability that the RI and eRBF values show abnormalities (small open circles) increases during the progression from Group A (G-A) through Group B (G-B)/Group C (G-C) and Group D (G-D) to Group $\mathrm{E}(\mathrm{G}-\mathrm{E})$. In contrast, the probability of normal values (large solid circles) decreases with the progression.

\section{Discussion}

\section{History of T2DN}

Kimmelstiel et al. have reported on characteristic glomerular lesions from the pathomorphological viewpoint in $1936^{13)}$. Wilson et al. have reported on glomerular lesions from the clinical viewpoint in $1951^{14)}$. Furthermore, functional and morphological abnormalities in early glomerular lesions have been reported by Hirose et al. in $1980^{15}$. As in these reports, T2DN is regarded as microangiopathy caused by functional and morphological impairments in glomeruli 




Relative frequency of normal and abnormal subjects undergoing ultrasound measurement. $\bigcirc$ abnormal

normal

Figure 6.
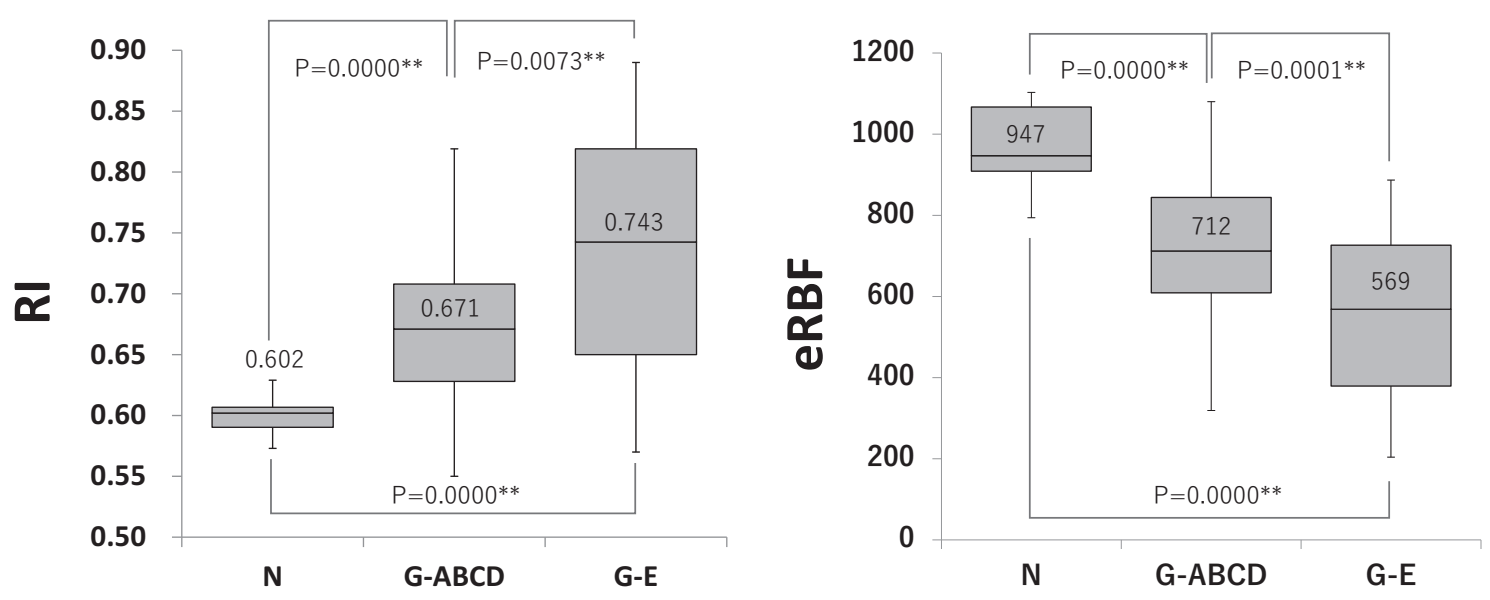

Intergroup Comparison of Renal Arterial Resistive Index (RI) and Estimated Renal Blood Flow (eRBF) $\mathrm{N}$, Normal healthy group; G-ABCD, urinary protein negative MetS group; $G-E$, urinary protein positive MetS group

Figure 7.

and arterioles, and this view continues up to today. These investigators also have pointed out the coexistence of intrarenal arteriosclerotic lesions. The question why these two morbid states coexist has arisen, but it has remained to be solved. A concept of MetS-N with intrarenal arteriosclerotic lesions will provide the answer to that question.

\section{Relationship between T2DM and MetS}

First, 465 T2DM patients with a proteinuria negative finding were reviewed for the participation of MetS in retrospective analysis. The participation accounted for $40 \%$ of the total in the cross-sectional analysis conducted at the time of physical examination and as high as $74.2 \%$ in the longitudinal analysis (in which patients had been examined for the past clinical course in detail, i.e., it was demonstrated that three in four T2DM patients had MetS (Figure 4). Second, 77 individuals with obesity alone at the first year of the physical examination, started in year 2008, were followed up solely under the lifestyle guidance through prospective analysis for 5 years. As shown in Figure 5, this analysis disclosed that risk factors for MetS appeared sequentially. More importantly, it was shown that, once the four risk fac- tors for MetS became overlapped, individuals with MetS$\mathrm{N}$-having a proteinuria positive finding increased in number gradually. This was considered to be a combined state of intrarenal arteriosclerotic lesions and microangiopathy as an outgrowth of the MetS. These findings suggest that fullhouse MetS-N almost comprehensively covers T2DN, benign nephrosclerosis, and obesity-related nephropathy as distinct clinicopathological entities (Figure 8).

\section{Surrogate Markers of Intrarenal Arteriosclerosis}

Concerning a relationship between RI and eRBF as assumed surrogate markers of intrarenal arteriosclerosis, when the proteinuria negative group (Groups A to D), the proteinuria positive group (Group E), and normal healthy controls (N) are compared with one another, the RI and eRBF for Groups A to D and Group E showed gradual aggravation while presenting a mirror image of each other (Figure 6). This finding suggests that RI and eRBF can serve as surrogate markers of intrarenal arteriosclerosis. To date, there have been few reports on renal pathological characteristics ${ }^{16)}$ contrasted with RI and eRBF, therefore further studies are needed to collect case information. The use of eRBF at this 


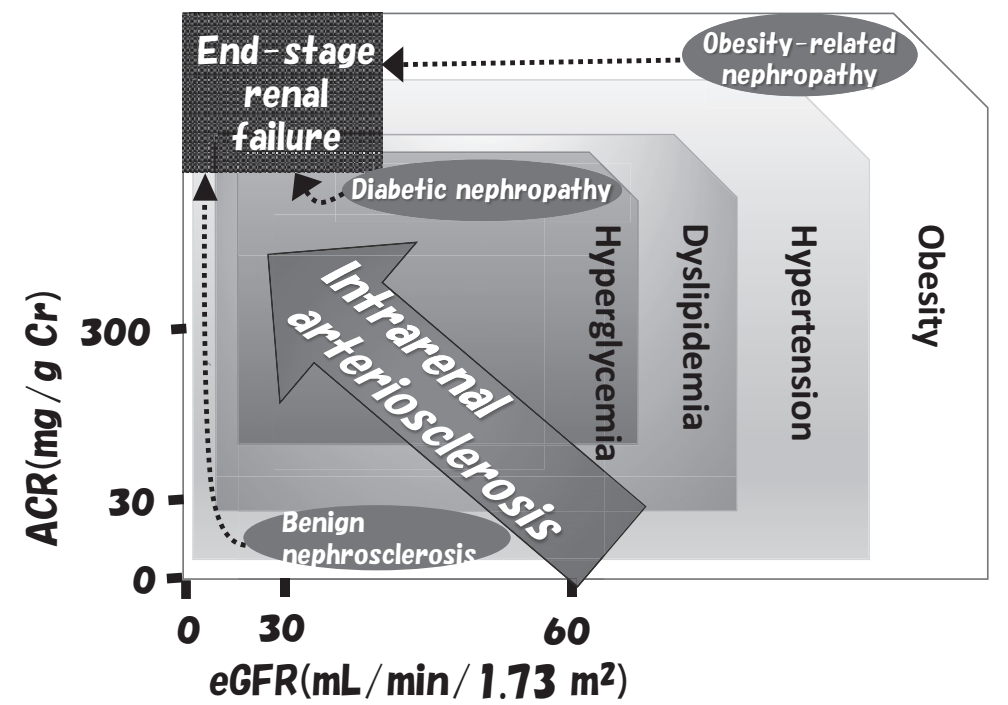

Clinical Course of Metabolic Syndrome Nephropathy (MetS-N): Obesity progresses from hypertension through dyslipidemia to hyperglycemia and, during this progress, microvascular damage and intrarenal arteriosclerosis emerge, leading to end-stage renal failure.

Figure 8.

\section{Relationship among Metabolic Syndrome Nephropathy, Diabetic Kidney Disease, \\ and Diabetic Nephropathy}

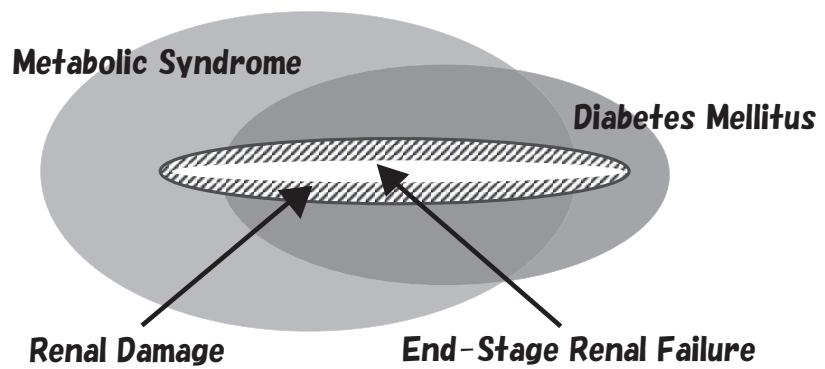

Figure 9.

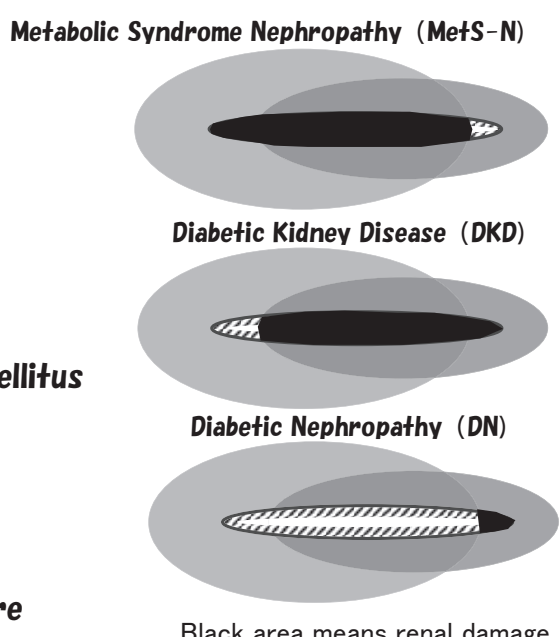

Black area means renal damage. purport is the world's first attempt. With respect to the present pilot study, therefore, a comparative evaluation of two different approaches- $\mathrm{eRBF}$ and the renal blood flow measured by the standard renal clearance procedure using paraaminohippuric acid-is needed.

\section{Progressive Pattern of MetS-N}

As illustrated in Figure 8, MetS begins with obesity, and then proceeds from hypertension to dyslipidemia and hyperglycemia. During this progressive course, intrarenal arteriosclerosis occurs before one is aware. It can be presumed that the arteriosclerosis, especially intimal thickening (fibroelastosis) of the arcuate artery, gradually extends the area. Nevertheless, it is difficult to demonstrate the progression of such pathological features in detail. Therefore, RI and eRBF as surrogate markers will be useful in presuming the relevant pathological features. Of course, glomerular damage peculiar to T2DN( nodular lesion, diffuse lesion, and exudative lesion) and arteriolosclerosis as microangiopathy appears according to the time of onset and severity of hyperglycemia. In confirmation of these views, it will be useful to accumulate a lot of information from the patients nephrectomized for kidney cancer who present pathological features of the entire kidney ${ }^{16}$.

\section{Relationship among MetS-N, Diabetic Kidney Dis- ease (DKD), and DN}

The relationship among T2DN, recent high-visibility DKD, and MetS-N will be discussed below. MetS is very closely related to T2DM because, as stated above, they overlap at a rate of $74.2 \%$. Figure 9 demonstrates how the occurrence of renal damage (diagonal area) in part in each of MetS, DKD, and T2DM leads to end-stage renal failure (open area). Each renal damage in MetS, DKD, and T2DM 
is illustrated by the top right figure, middle right figure, and bottom right figure, respectively. When preventive strategies for end-stage renal failure are taken on the basis of the present situation under which T2DM patients with MetS are on the increase and dialysis patients are not on the decrease, it may be said that this schema is highly suggestive. Why do MetS patients with the proteinuria negative T2DM, especially Group A, have such a high incidence of renal damage? This question may be explained as follows: obesity, hypertension, and dyslipidemia are intricately linked with one another for long periods of time; at the onset of T2DM, intrarenal arteriosclerotic lesions are already present and ageing has also added. Thus, most of MetS-N followed by early optimal strategies will allow a marked reduction in the number of patients with end-stage renal failure.

\section{Limitation}

(1) We retrospectively screened clinical history and laboratory information for T2DM patients who underwent daily medical practice. This suggests that the information may lack precision. On that point, the relevant information for 77 individuals solely with obesity pointed out under the specified medical checkup/health guidance has high precision because they were prospectively observed.

(2) Information concerning $\mathrm{RI}$ and $\mathrm{eRBF}$ as surrogate markers of intrarenal atherosclerosis is scanty. Nevertheless, RI has been building up a reputation for its capability, whereas further studies are needed to use eRBF as a surrogate marker because the present study is the world's first trial.

(3) The onset form of MetS-N is nothing short of ignorance at present. In view of the author's observations, however, intrarenal atherosclerotic lesions, in addition to the microangiopathy observed after the onset of type 2 diabetes mellitus, are supposed to have already been caused at the onset of type 2 diabetes mellitus. In other words, it is, at this time, difficult to clarify whether microangiopathy and intrarenal atherosclerotic lesions occur individually or their onset is in line with each other. The author believes the latter, however.

\section{Conclusion}

We named the nephropathy with full-house MetS "MetSN". A new method using RI and eRBF that may have relation to intrarenal arteriosclerotic lesions, as compared with the conventional method using ACR and eGFR, can detect early state of MetS-N at a high rate. The new method-based diagnosis followed by opitmal strategies can contribute greatly to the prevention of end-stage renal failure.

Conflicts of Interest

Non-contributory.

\section{Acknowledgments}

Thanks are tendered to Dr. Kazumasa Aoyagi, Dr. Hiroo Niimi, Dr. Yasumi Uchida, Dr. Hiromi Isikawa, Mr. Hideyuki Higaki, Ms. Yoko Tsuchiya, and Toshio Hiroi for their long painstaking collaboration.

\section{References}

1. Japanese Society for Dialysis Therapy Committee of Renal Data Registry. ILLUSTRATION Current status of chronic dialysis therapy in Japan (as of 31 December 2016). 2016.

2. Gaede P, Oellgaard J, Carstensen B, Rossing P, Lund-Andersen H, Parving HH. Years of life gained by multifactorial intervention in patients with type 2 diabetes mellitus and microalbuminuria: 21 years follow-up on the Steno-2 randomised trial. Diabetologia 2016; 59: 2298-307.

3. Ueki K, Sasako T, Okazaki Y, Kato M, Okahata S, Katsuyama H, et al. Effect of an intensified multifactorial intervention on cardiovascular outcomes and mortality in type 2 diabetes (J-DOIT3): An open-label, randomized controlled trial. Diabetes-Endocrinology 2017; 24: 30327-3

4. Tsuchida H. Preventive strategy for end-stage-renal-failure: A proposal of metabolic-syndrome-nephropathy on the point of highschool-urinalysis in Chiba prefecture for 20 years. Chiba Med J 2012; 88: 11-25.

5. Tsuchida H. [TOPICS] Metabolic syndrome nephropathy-Its clinicopathological features and early diagnosis. Kidney and Dialysis 2015; 78(4): 605-10.

6. Committee on Diagnostic Criteria for Metabolic Syndrome. Definition and diagnosis criteria of metabolic syndrome. J Jpn Soc Intern Med 2005; 94: 794-809.

7. Mogensen CE. N Engl J Med 1984; 310: 356-60.

8. Matsuo S, Imai E, Horio M, Yasuda Y, Tomita K, Nitta K, et al. Revised equations for estimated GFR from serum creatinine in Japan. Am J Kidney Dis 2009; 53: 982-92.

9. Takeyama S, Iwashita K, Yamaguchi H, Sato T. Renal ultrasonography of diabetic nephropathy--Renal hemodynamic evaluation by pulse Doppler method. Jpn J Med Ultrasound Technol 2007; 27: 5-9.

10. Iwashita $\mathrm{K}$, Tsuchida $\mathrm{H}$. Consideration of physiological examination and laboratory testing: symposium. Rinsho Byori 2014; 62: 1104-9.

11. Tsubaki T. Basic study on renal arterial blood flow (segmental arteries and interlobar arteries) measurement by an ultrasonic Doppler method in normal individuals. Jpn J Med Technol 2003; 52: 8.

12. Haneda K, Utsunomiya K, Koya D, Babazono T, Moriya T, Makino H, et al. Classification of Diabetic Nephropathy 2014. Jpn J Nephrol 2014; 56: 547-52.

13. Kimmelstiel P, Wilson C. Intercapillary lesions in the glomeruli of the kidney. Am J Path 1936; 12: 83-97.

14. Wilson JL, Root HF, Marble A. Diabetic nephropathy-A clinical syndrome. N Engl J Med 1951; 245: 513-7.

15. Hirose K, Tsuchida H, Osterby R, Gundersen HJ. A strong correlation between glomerular filtration rete and filtration surface in diabetic kidney hyperfunction. Lab Invest 1980; 43(5): 434-7.

16. Alexander MP, Patel TV, Farag YM, Florez A, Rennke HG, Singh AK. Kidney pathological changes in metabolic syndrome. Am J Kidney Dis 2009; 53: 751-9. 